\title{
Interaction of a Tin-based capillary porous structure with ITER/DEMO relevant plasma conditions
}

\author{
T. W. Morgan ${ }^{\mathrm{a}^{*}}$, D. C. M. van den Bekerom ${ }^{\mathrm{a}}$ and G. De Temmerman ${ }^{\mathrm{a}}$ \\ FOM Institute DIFFER, Dutch Institute For Fundamental Energy Research, Ass. EURATOM-FOM, \\ Trilateral Euregio Cluster, Nieuwegein, The Netherlands
}

\begin{abstract}
Sn filled Capillary Porous Structures were exposed to high flux low temperature plasma conditions at the Pilot-PSI linear device. Enhanced erosion above that expected classically was investigated via spectroscopic observation of $\mathrm{Sn}^{0}$ emission from the plasma in front of the target surface while the surface temperature was monitored by both thermography and pyrometry. An anomalous erosion flux was observed as temperature increases, with onset for this occurrence varying strongly between different ion species. The results appear incompatible with existing 'adatom' models for the anomalous erosion flux. Further targets were exposed in turn to increasing heat fluxes and the heat removed determined from cooling water calorimetry, which was then compared to a solid Mo reference target. At high powers the total energy of the cooling water is reduced, indicating a shielding of the surface from the plasma heat flux by the vapour cloud in front.
\end{abstract}

PACS: 52.40.Hf; 52.70.Nc; 52.25.Vy

PSI-20 keywords: Erosion \& Deposition; Liquid metal; Power handling; Spectroscopy; Sputtering

*Corresponding/presenting author address: Edisonbaan 14, Nieuwegein, The Netherlands

*Corresponding/presenting author E-mail:t.w.morgan@differ.nl 


\section{Introduction}

Due to the high power loads and neutron effects in a future fusion reactor the performance of solid plasma facing materials (PFMs) may prove to be insufficient for the operational regime required. Liquid metals (LMs) offer the possibility of a "self healing" surface where erosion and damage is replaced through new material flowing in, the potential for improved heat removal through combining convection and conduction, as well as inherent immunity to neutron embrittlement. Currently Li, Sn and Ga are the main candidate materials [1]. Sn and Ga in particular offer the possibility of using a high recycling, low tritium-retention surface.

Many experiments [2-7] have reported an enhancement of the erosion yield of materials under plasma bombardment as a function of temperature above that expected from physical sputtering and evaporation alone. If migration of material eroded from an LM surface by the plasma into the core of the tokamak becomes too large, this could lead to large-scale cooling of the plasma leading to the extinguishing of the fusion reaction. This may therefore limit the thermal operating window of the surface. However, the incoming plasma pressure may limit the liquid metal ions to the near surface region, which would lead to localised radiative cooling and very high re-deposition rates onto the LM surface, both of which would be beneficial to the power handling capabilities of the LM divertor. Therefore it is important to generate a greater understanding of the dynamics of the region of the plasma close to the liquid surface and the interaction between the two.

\section{Experimental Set-up}

Experiments were conducted in the Pilot-PSI linear device (fig 1a). A cascaded arc source [8,9] generates the plasma which is confined by a variable magnetic field (0.2-1.6 T) into a high flux plasma beam. The machine can use a wide variety of source gases $(\mathrm{H}, \mathrm{D}, \mathrm{He}, \mathrm{N}, \mathrm{Ne}, \mathrm{Ar})$ and is capable of producing high flux, low temperature conditions similar to those expected in the 
divertor of ITER or DEMO. Typical plasma parameters used here are $\mathrm{n}_{\mathrm{e}} \sim 10^{20-21} \mathrm{~m}^{-3}, \mathrm{~T}_{\mathrm{e}} \sim 0.5-$ $2 \mathrm{eV}, \Gamma \sim 10^{23-24} \mathrm{~m}^{-2} \mathrm{~s}^{-1}, \mathrm{q} \sim 1-15 \mathrm{MW} \mathrm{m} \mathrm{m}^{-2}$

Target containers constructed of TZM molybdenum were filled with a stack of W or Mo meshes with pore diameter between 25 and $100 \mu \mathrm{m}$ to create a static Capillary Porous Structure (CPS). $\mathrm{W}$ and Mo were chosen both for their good material properties as plasma facing components and due to chemical and wetting compatibility with Sn [1]. These were cleaned in an acetone sonic bath and filled with $\mathrm{Sn}$ in a vacuum oven which heated the cups to above $800{ }^{\circ} \mathrm{C}$ to facilitate wetting. The CPS acts to confine the liquid Sn during plasma exposure from motion and leaking by capillary forces acting against hydrostatic, plasma pressure and $\boldsymbol{j} \mathrm{x} \boldsymbol{B}$ forces [10] (fig 1b).

The region in front of the target surface at the beam centre $(r=0)$ was observed by a singlechannel wide spectrum spectrometer (Avantes ULS2048 299nm to $579 \mathrm{~nm}$ ) to monitor emission from the vapour cloud and plasma in front of the mesh, while the temperature of the liquid surface was monitored using both a multicolour pyrometer (FMPI SpectroPyrometer), which also observed at $r=0$, and an infra-red camera (FLIR A645 sc). The vapour plume was monitored using a fast visible camera (Phantom V12) with a $452.5 \pm 1 \mathrm{~nm} \mathrm{Sn}^{0}$ filter viewing tangentially to the plasma beam. The energy deposited on the targets was determined from calorimetry of the target cooling water, while electron temperature and density of the plasma was ascertained from Thomson scattering made while a reference Mo target was used at identical settings, as $\mathrm{Sn}$ in the plasma caused excessive stray light.

\section{Measurements}

\subsection{Temperature enhanced erosion}

\subsubsection{Theory}


Plasma can classically erode surfaces in one of two possible ways: via sputtering, which is dependent only on incoming flux $\left(\Gamma_{i n}\right)$ and ion energy and species (i.e. yield, $Y_{p s}$ ), and evaporation $\left(\Gamma_{\text {evap }}\right)$, which at low pressures depends only on surface temperature $\left(T_{s}\right)$. We also define an anomalous flux $\left(\Gamma_{\text {anom }}\right)$ which represents any increased erosion measured above the classical predictions. The resultant erosion flux (particles $\mathrm{s}^{-1}$ ) is then either ionised in the plasma beam $\left(\Gamma_{i o n}\right)$ or lost from the plasma without ionisation $\left(\Gamma_{l o s s}\right)$, i.e.

$$
\Gamma\left(E_{\text {ion }}, T_{s}\right)=\Gamma_{i n} Y_{p s}\left(E_{\text {ion }}\right)+\Gamma_{\text {evap }}\left(T_{s}\right)+\Gamma_{\text {anom }}=\Gamma_{\text {ion }}+\Gamma_{\text {loss }}
$$

If $\Gamma_{\text {loss }}$ is negligible then we can relate the line-of-sight integrated intensity of eroded Sn neutrals $I_{S n 0}\left(\right.$ photons $\mathrm{sr}^{-1} \mathrm{~s}^{-1}$ ) to the erosion flux as [11]

$$
\Gamma=4 \pi \frac{S\left(T_{e}\right)}{X\left(T_{e}\right) B} I_{S n 0}
$$

where $S / X B$ is the so-called ionisation per photon. While many values for this are available in the literature [12], for Sn this is not the case so we must quantify the relationship between erosion and line intensity experimentally. This is done for low temperatures $\left(\Gamma_{\text {evap }}+\Gamma_{\text {anom }} \sim 0\right)$, so that $Y_{\text {meas }}=A_{\lambda}\left(T_{e}\right) N_{S n o} / \Gamma_{\text {in }}$, where $A_{\lambda}$ is a constant to be found for a specific excitation of wavelength $\lambda$ and $N_{S n O}$ are the (background subtracted) counts per second measured by the spectrometer for the chosen emission line. By using $N_{S n} 0$ uncertainties in determining the absolute integrated line intensity are avoided as $I_{S n} \propto \propto N S n 0$ for a given line of sight. By varying the bias voltage at constant electron temperature $Y_{\text {meas }}$ for different ion energies is determined and, given the assumption that binary-collision approximation calculations of sputtering yield using TRIM [13] are correct, these can be compared with $Y_{\text {TRIM }}$ to find the constant $A_{\lambda}$. It should be noted that this constant is then valid for all ion species.

\subsubsection{Results}


As in [14] the lost fraction can be estimated as $\Gamma_{\text {loss }} / \Gamma=\exp \left(-L / \lambda_{m f p}\right)$ where $L$ is the characteristic length of the system, defined here as the plasma beam HWHM $(\sim 5 \mathrm{~mm})$ and $\lambda_{m f p}$ is the ionisation mean free path of the neutrals. Observations using the filtered fast-visible camera viewing tangentially to the plasma beam at the beam centre were made using a hydrogen plasma with a density of $1 \times 10^{20} \mathrm{~m}^{-3}$ and electron temperature of $0.5 \mathrm{eV}$ at $-30 \mathrm{~V}$ bias, which gives a lower bound for typical densities and temperatures (which are otherwise in this work higher) and thus an upper limit on $\lambda_{m f p}$. This is determined from the line-integrated intensity at $r=0$ as $I_{\text {int }}=B \exp (-$ $z\left(\lambda_{m f p}\right)+C$, where $z$ is the distance along the plasma beam axis from the target and $\mathrm{B}$ and $\mathrm{C}$ are constants to be fitted (figure 2). From this we find $\lambda_{m f p}=2 \pm 0.3 \mathrm{~mm}$, giving $\Gamma_{\text {loss }} / \Gamma \leq 0.08 \pm$ 0.03 which indicates our above assumptions are reasonable.

$Y_{\text {meas }}$ for $\mathrm{Ar}$ on $\mathrm{Sn}$ is compared to $Y_{\text {TRIM }}$ in figure 3 and shows excellent agreement for a wide number of $\mathrm{Sn}^{0}$ emission lines. From this $A \lambda$ can be determined for each line. The $317.50 \mathrm{~nm} \mathrm{Sn}$ line was chosen from this point as it is a strong transition from a metastable ground state in the observed wavelength region free from interfering lines from our ion species [15], and $A 317.50$ is found to be $4 \times 10^{16}$ particles count ${ }^{-1}$.

From this erosion fluxes were determined as a function of temperature for a variety of biases for the species $\mathrm{H}, \mathrm{He}$ and $\mathrm{Ar}$ (figure 4). Expected evaporation fluxes [16] and predictions of physical sputtering [13] are shown also.

\subsection{Power handling}

As the loss fraction of tin neutrals is estimated to be low $(<8 \%)$ a large amount of eroded tin remains in the plasma beam where it is excited and ionised, before being entrained by the plasma ions and re-deposited back on the surface of the target. This vapour layer may shield the surface from some of the plasma heat flux by radiation, resulting in a lower heat flux to the target. While a vapour cloud is predicted by modelling [17] and supported by some 
experimental results which indicate shielding of the surface to have occurred $[18,19]$ it is currently unclear as to its efficacy in improving divertor performance, and whether it will stably remain in front of the liquid surface on longer time scales. Therefore it is important to generate a greater understanding of the dynamics of the region of the plasma close to the liquid surface and the interaction between the two.

To investigate this Sn targets were exposed to six discharges of increasing power and density (from $\mathrm{n}_{\mathrm{e}} \sim 1 \rightarrow 15 \times 10^{20} \mathrm{~m}^{-3}$ ) and the total removed energy determined from calorimetry of the target cooling water. This was compared to a reference solid Mo target. These discharges are summarised in figure 5. These show that at higher discharge powers the heat flux to the target is indeed reduced compared to the reference. As the energy was not absorbed by the target it must have been lost from the plasma before reaching the surface which provides evidence that vapour shielding is taking place at high fluxes and densities.

\section{Discussion}

The values measured for erosion flux cannot be explained from physical sputtering and evaporation alone. Due to the high re-deposition fraction some increase of signal may possibly be due to self-sputtering by $\mathrm{Sn}$, which can thus liberate more $\mathrm{Sn}$ in an ongoing cycle. This increases the expected erosion flux as a geometric series by a factor $\Gamma /\left(1-Y_{S n}\right)$ if redeposition is $100 \%$. However, even for the highest bias applied in these experiments $(-80 \mathrm{~V})$ the yield predicted from TRIM [Behrisch and Eckstein] is only 0.128, giving an increase of $<15 \%$, while the anomalous erosion is found even for biases of $-30 \mathrm{~V}$ where the effect would be of the order $0.4 \%\left(Y_{S n} \sim 0.004\right)$. Although $\mathrm{Sn}$ ions could be entrained and accelerated to the plasma sound speed, the fact they are confined close to the target surface indicates they would strike the wall before reaching high velocities [20]. Furthermore this does not fit with other experiments where re-deposition was negligible. 
A second possibility suggested by Doerner et al. [21] proposes the creation of adatoms, where incoming particle bombardment leads to creation of a surface vacancy and relocation of a surface particle onto the surface plane, where it is bound with a lower energy and can thus sublimate more easily than an evaporated particle. Thus it is dependent both on flux and surface temperature and should (like evaporation) follow an Arrhenius relationship of the form

$$
\Gamma_{\text {anom }}=\Gamma_{i n} Y_{E F F} \exp \left(-\frac{E_{E F F}}{k_{B} T_{s}}\right)
$$

Where $Y_{E F F}$ is the effective yield based on the areal density and lifetime of the adatoms and $E_{E F F}$ is the effective adatom sublimation energy. For $\mathrm{Sn}$ the surface binding energy is $3.08 \mathrm{eV}$. Fitting Arrhenius curves to the anomalous data gives $E_{E F F}$ as $1.22 \mathrm{eV}$ for $\mathrm{Ar}, 1.50 \mathrm{eV}$ for $\mathrm{He}$ and $0.27 \mathrm{eV}$ for $\mathrm{H}$. This wide variation in $E_{E F F}$ suggests that this model is not a good fit for these results as these should not vary based on ion species. Neither this nor self-sputtering therefore appear to adequately explain the observed results.

In each case the measured erosion flux shows a marked increase above classical expectations due to an anomalous erosion process. This data fits with earlier results on Sn by Coventry et al. $[6,7]$ which also shows a differing temperature onset for anomalous erosion for differing ion species. In their case the onsets for increased erosion were $>700{ }^{\circ} \mathrm{C},>500{ }^{\circ} \mathrm{C}$ and $>300{ }^{\circ} \mathrm{C}$ for Ar, He and D ions at $1000 \mathrm{eV}$ and fluxes between $10^{17}$ and $10^{18}$ particles $\mathrm{m}^{-2} \mathrm{~s}^{-1}$. This gives an offset of $\sim 100{ }^{\circ} \mathrm{C}, \sim 200{ }^{\circ} \mathrm{C}$ and $\sim 350{ }^{\circ} \mathrm{C}$ for the temperature to reach the point for evaporation to match their measured erosion yields. For these results the offset is $\sim 200{ }^{\circ} \mathrm{C}, \sim 100{ }^{\circ} \mathrm{C}$ and $\sim 500{ }^{\circ} \mathrm{C}$ respectively for Ar, $\mathrm{He}$ and $\mathrm{H}$. These results thus show somewhat similar results in both cases, particularly the large temperature offset for H/D in each case. This suggests that the anomalous erosion, at least for tin, is a flux-independent phenomenon and that ion species or mass plays an important role in the erosion level. In particular the large difference both in 
temperature offset and in $E_{E F F}$ suggests a different behaviour for $\mathrm{H}$ compared to the two noble gases. This may indicate that chemical erosion, possibly through the production of stannane, plays an important role in this case, although the standard enthalpy of formation is strongly positive $(+1.68 \mathrm{eV}$ per atom [23]).

\section{Conclusions}

Experiments were performed at the Pilot-PSI linear device using Sn filled CPS targets to investigate the erosion yield and power handling of the tin surface. Erosion measurements show an anomalous erosion above classical expectations, which differs strongly dependent upon ion species. Fitting to the 'adatom' model of erosion does not fit well physically with these results. At high power loads a reduction of heat flux to the target as observed from calorimetry is found. This suggests that the eroded vapour cloud removes some power from the plasma before it reaches the target surface.

\section{Acknowledgements}

This work is part of the research program of FOM, which is financially supported by NWO. It is supported by the European Communities under the contract of Association between EURATOM and FOM.

\section{References}

[1] J.W. Coenen et al. Phys. Scr. T159 (2014) 014037

[2] J. Roth and W. Moller Nucl. Instrum. Methods Phys. Res. B 7-8 (1985) 788-792

[3] R. P. Doerner, M. J. Baldwin, D. G. Whyte, and S. Krasheninnikov, J. Nucl. Mater. 313 (2003) 383 
[4] R. W. Conn et al., Nucl. Fusion 42 (2002) 1060

[5] J. P. Allain, M. D. Coventry, and D. N. Ruzic, J. Nucl. Mater. 313 (2003) 641

[6] M.D. Coventry and D.N. Ruzic J. Nucl. Mater. 337-339 (2004) 1015-1018

[7] M.D. Coventry, J.P. Allain and D.N. Ruzic Proc. 21st SOFE Conference, Knoxville USA (2005)

[8] H. Z.Maecker Naturforsch. 11(A) (1956) 457

[9] M. C. M. van der Sanden et al. Rev. Sci. Instrum. 63 (1992) 3369

[10] V. A. Evtikhin et al. J. Nucl. Mater. 271-272 (1999) 396-400

[11] K. Behringer J. Nucl. Mater.145-147 (1987) 145-153

[12] H.P. Summers The ADAS User Manual, version 2.6 (http://www.adas.ac.uk/manual.php) (2004)

[13] R. Behrisch and W. Eckstein W Sputtering by Particle Bombardment (Berlin: SpringerVerlag) (2007)

[14] Nishijima et al. Phys. Plasmas 16 (2009) 122503

[15] A. Kramida et al. NIST Atomic Spectra Database (ver. 5.1) ( http://physics.nist.gov/asd) (2013)

[16] C. B. Alcock, V.P. Itkin, and Horrigan, M. K., Canadian Metallurgical Quarterly 23 (1984) 309

[17] A. Hassanein, T. Sizyuk and I. Konkashbaev, J. Nucl. Mater. 390-391 (2009) 777-780

[18] M. A. Bourham et al. Plasma Sci., IEEE Transactions (1989) 386-391

[19] S. Mirnov et al. Plasma Phys. Control. Fusion 48 (2006) 821-837

[20] G. Van Swaaij Studies of impurity transport in high density, low temperature plasma with the ERO code (http://alexandria.tue.nl/extra2/774191.pdf) (2014)

[21] R. P. Doerner, S. I. Krasheninnikov, and K. Schmid J. Appl. Phys. 95 (2004) 4471

[22] Y. Kudriavtsev, A. Villegas, A. Godines and R. Asomoza Appl. Surf. Sci. 239 (3-4) (2005) 273-278

[23] D. R. Lide CRC Handbook of Chemistry and Physics, 85th ed. (Boca Raton: CRC Press) (2005) 


\section{Figure captions}

1. (a) diagram showing the Pilot-PSI machine and diagnostics as used in this work (b) Sn target schematic showing dimensions and components

2. Line integrated intensity of $\mathrm{Sn}^{0} 452.5 \mathrm{~nm}$ light at $r=0$ as a function of axial distance $(z)$ from the target, as well as fit to the data of the form $I_{\text {int }}=B \exp \left(-z / \lambda_{m f p}\right)+C$

3. Comparison between expected yield values for Ar ions onto a Sn surface from TRIM and measured values $Y_{\text {meas }}=A_{\lambda}\left(T_{e}\right) N_{S n} 0 / \Gamma_{i n}$, where the constant $A_{\lambda}$ has been fitted for each Sn line shown.

4. Erosion flux of $\mathrm{Sn}$ by (a) $\mathrm{Ar}$ (b) $\mathrm{He}$ and (c) $\mathrm{H}$ ions as a function of ion energy (bias) and surface temperature. Expected evaporation curves and sputtering flux (Ar $-40 \mathrm{~V}, \mathrm{He}-$ $50 \mathrm{~V}$ ) are also shown, as well as the expected total flux including adatom sublimation using equation (3).

5. Removed heat as measured by calorimetry of the cooling water for six shots of increasing input power (A to F) as a function of input source power, for both a Mo reference target and a Sn filled target. 
Figure 1:

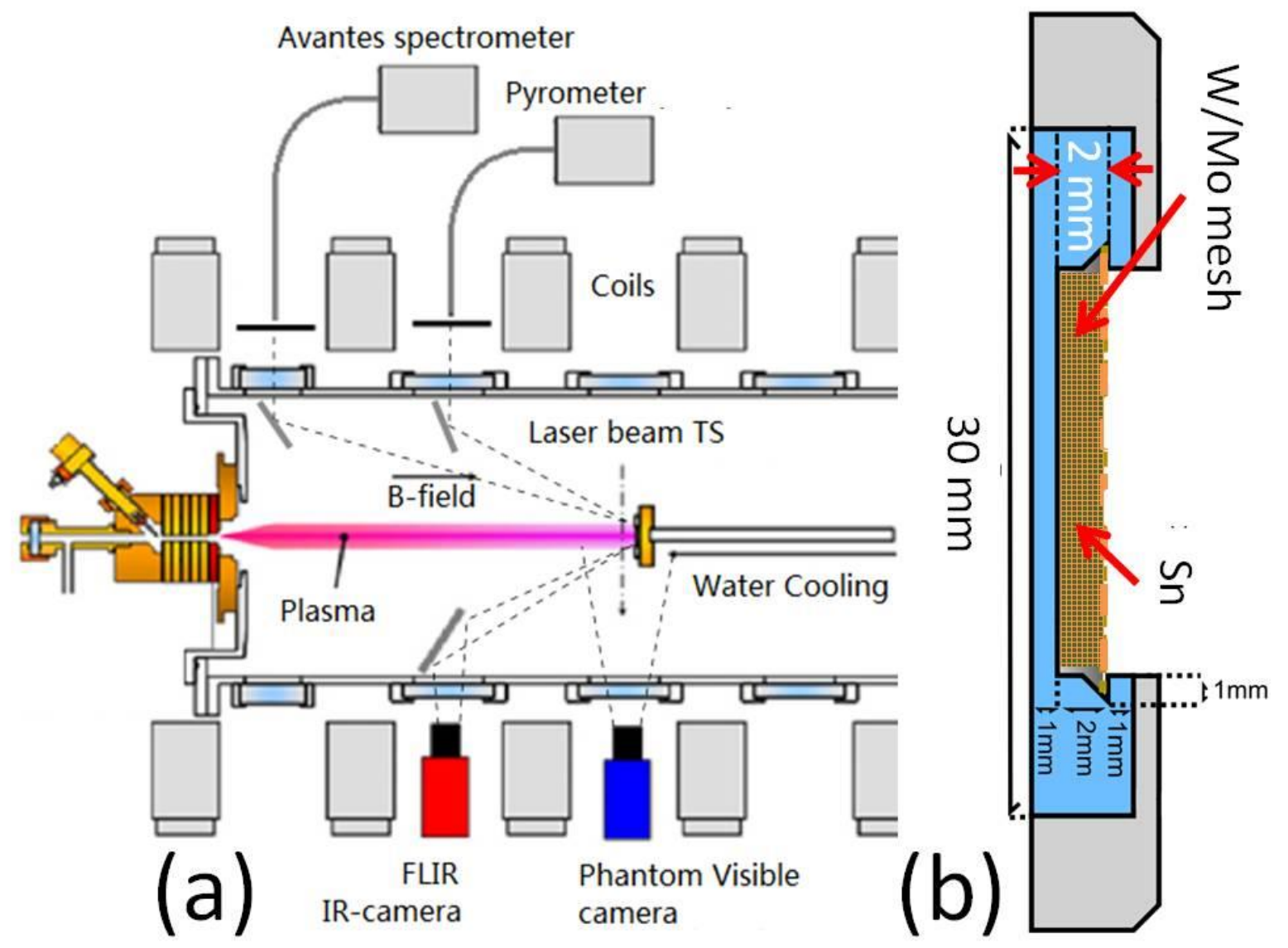


Figure 2:

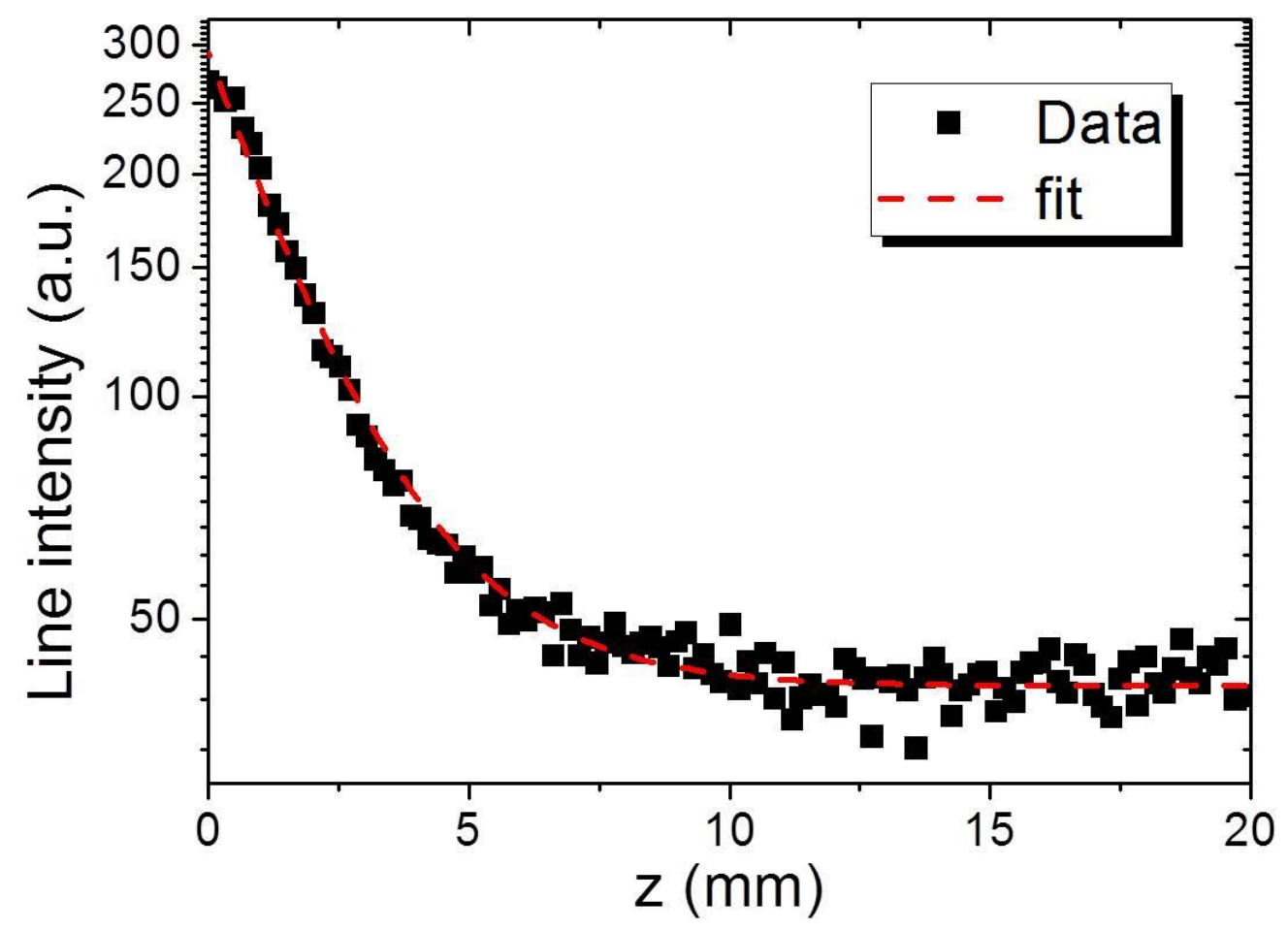


Figure 3:

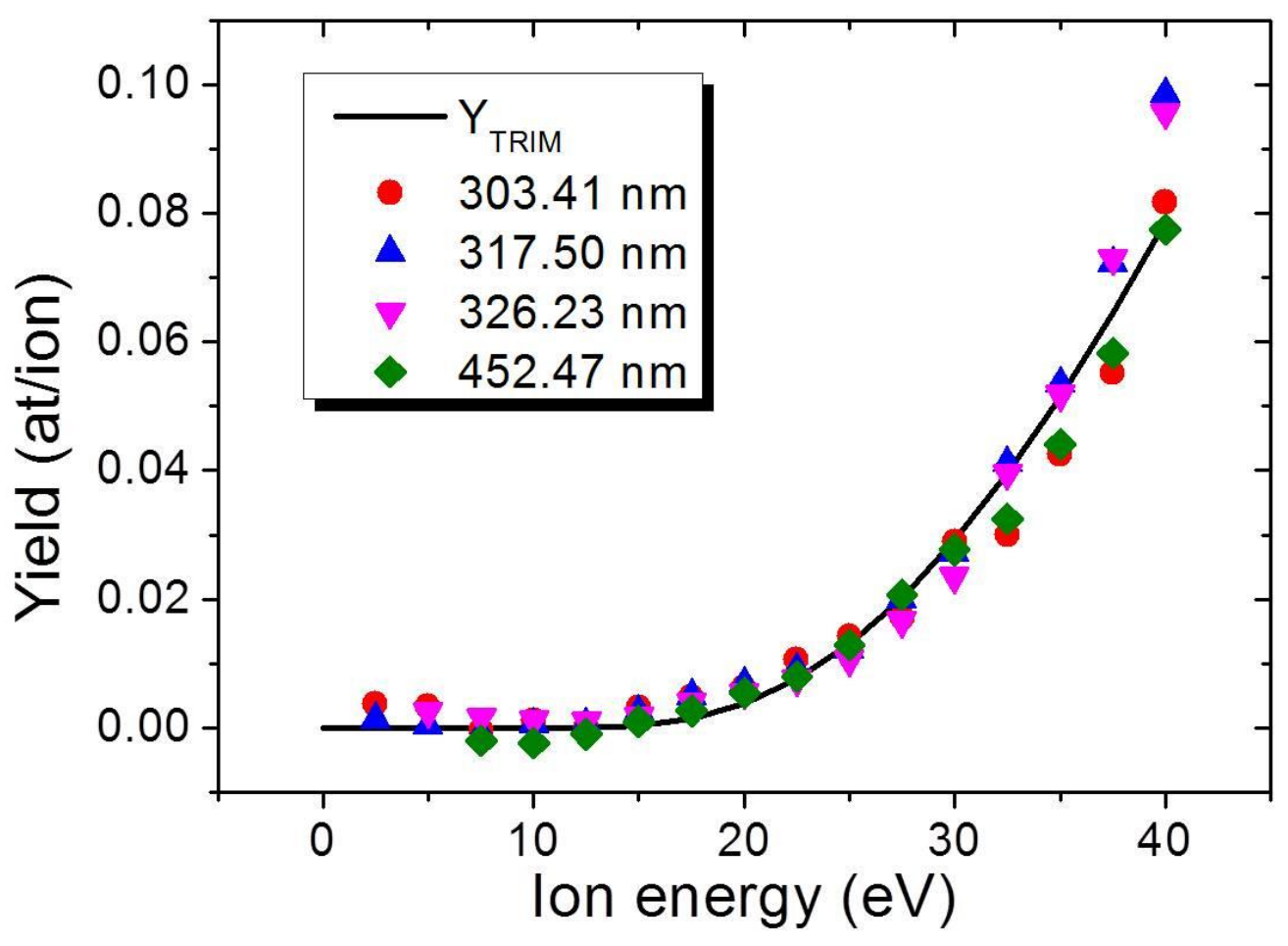



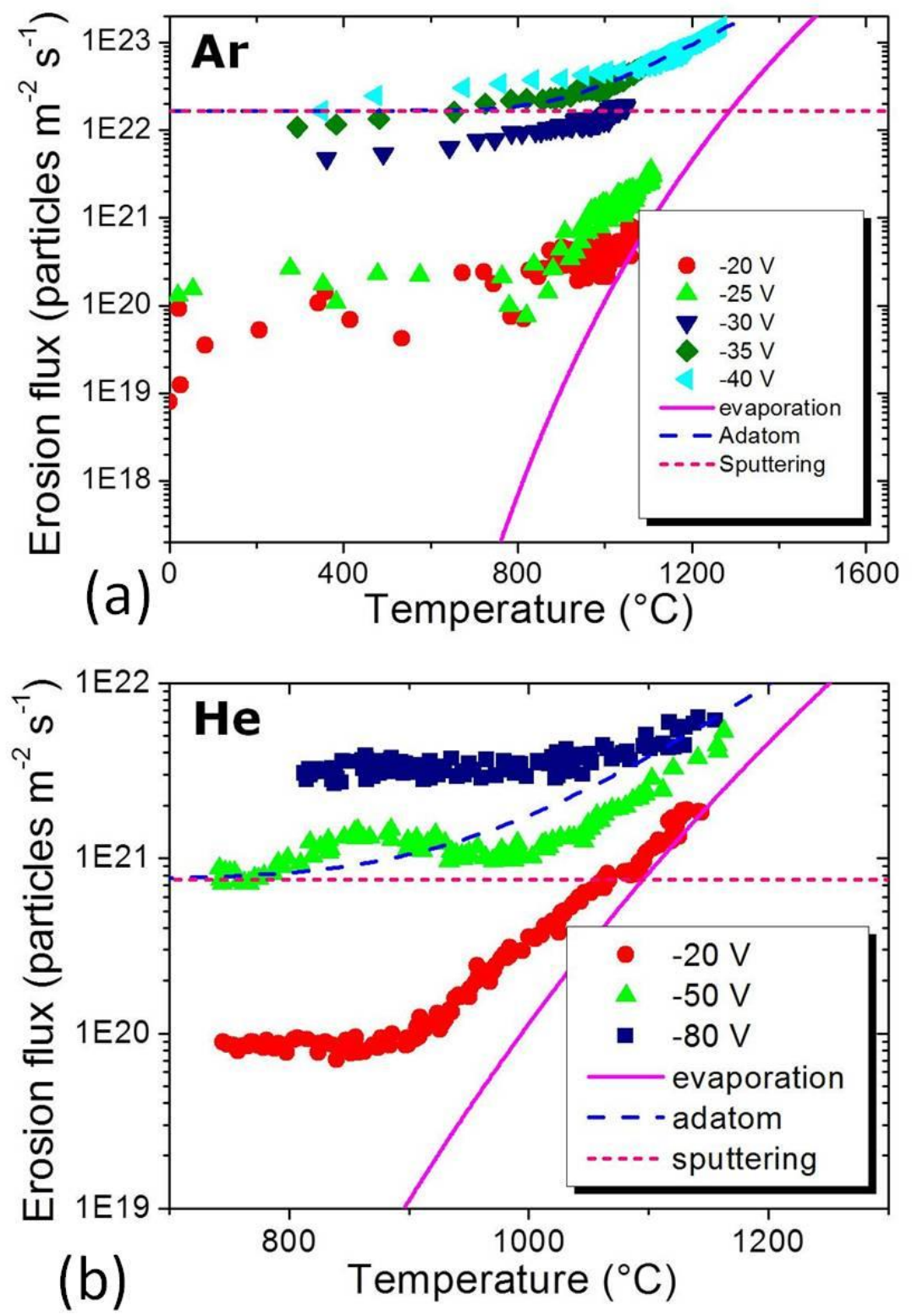

Figure 4:

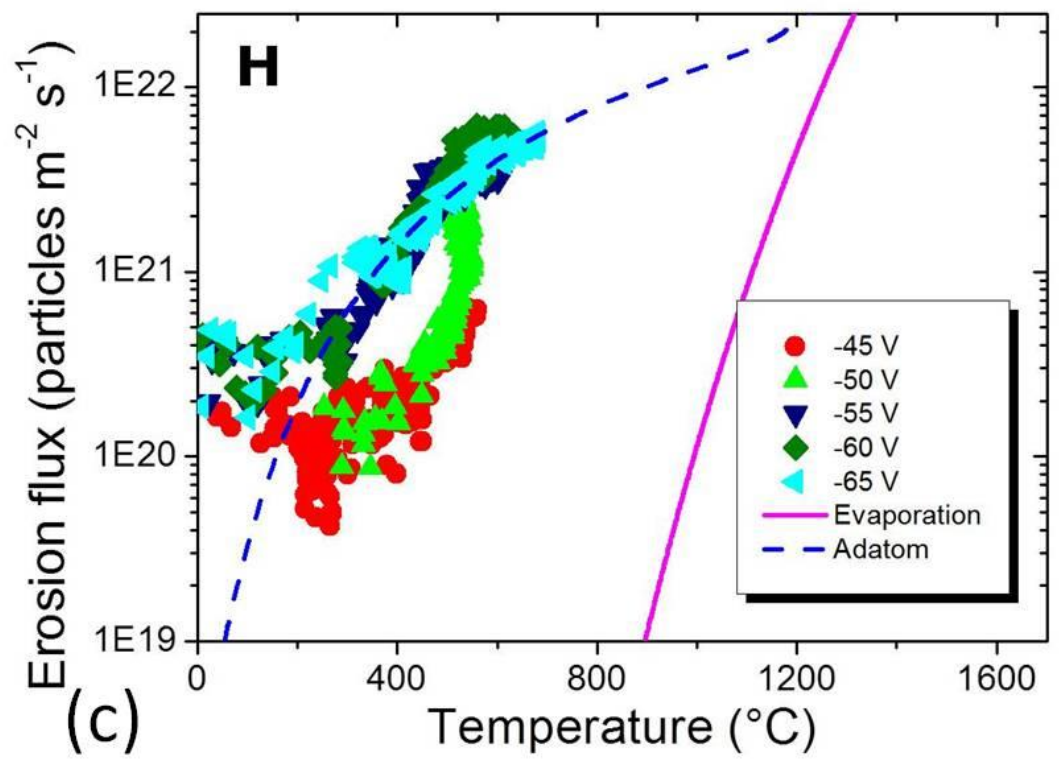


Figure 5:

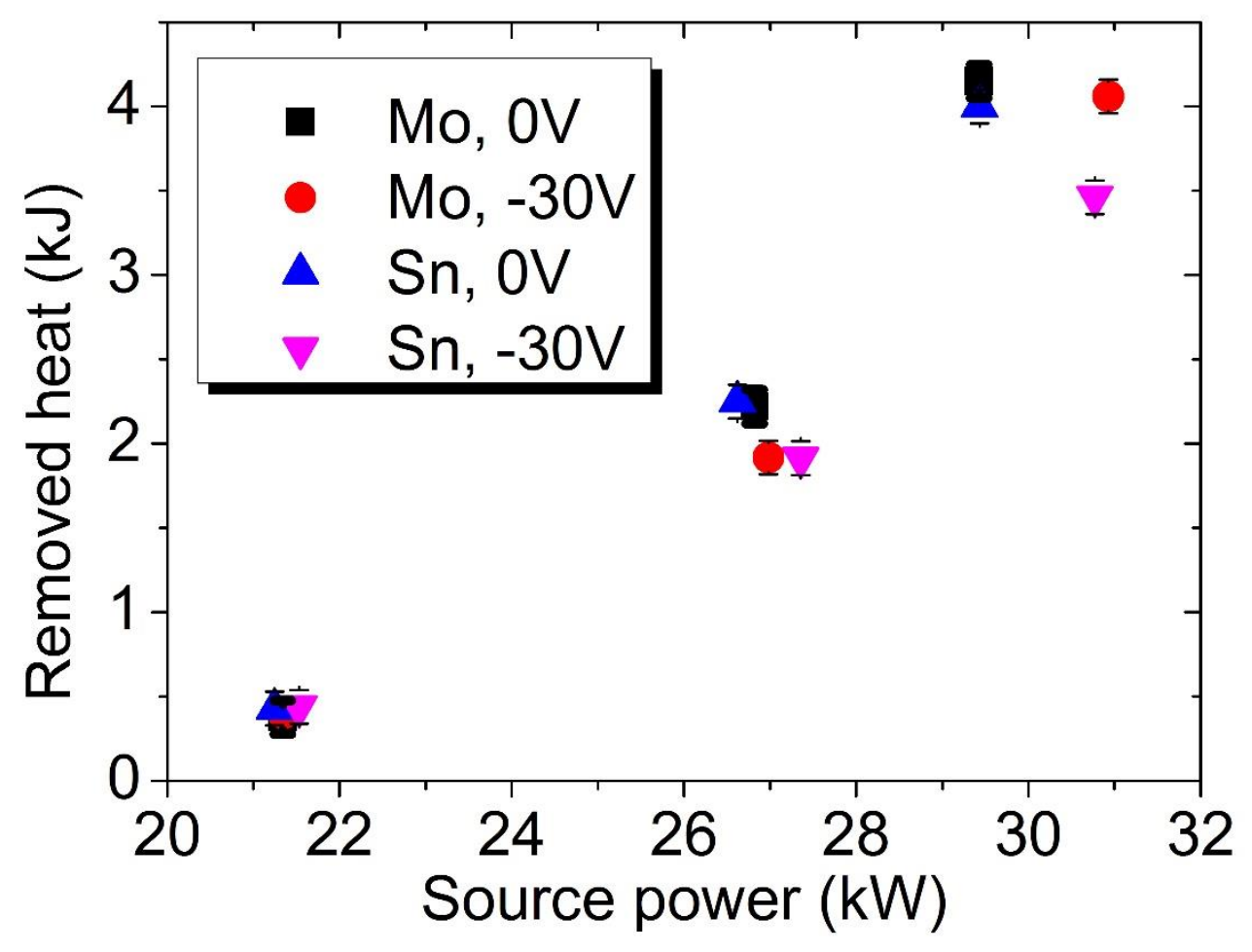

